\title{
Engineered miniaturized models of musculoskeletal diseases
}

\author{
Simone Bersini ${ }^{1}$, Chiara Arrigoni ${ }^{1, \dagger}$, Silvia Lopa ${ }^{1, \dagger}$, Matilde Bongio ${ }^{1, \dagger}$, Ivan \\ Martin $^{2}$, Matteo Moretti $i^{3,4,5,6}$
}

${ }^{1}$ Cell and Tissue Engineering Lab, IRCCS Istituto Ortopedico Galeazzi, Milano, Italy

${ }^{2}$ Department of Surgery and Department of Biomedicine, University Hospital Basel, University of Basel, 4031 Basel,

Switzerland

${ }^{3}$ Cell and Tissue Engineering Lab, IRCCS Istituto Ortopedico Galeazzi, Milano, Italy

${ }^{4}$ Regenerative Medicine Technologies Lab, Ente Ospedaliero Cantonale (EOC), Lugano, Switzerland

${ }^{5}$ Swiss Institute for Regenerative Medicine, Lugano, Switzerland

${ }^{6}$ Fondazione Cardiocentro Ticino, Lugano, Switzerland

Corresponding author: Moretti, M. (matteo.moretti@grupposandonato.it).

*These authors contributed equally.

Keywords: miniaturized; in vitro model; musculoskeletal; disease.

Teaser: In vitro human miniaturized models represent next-generation systems with which to study the mechanisms driving musculoskeletal diseases, identify novel biomarkers, and screen potential drug candidates.

The musculoskeletal system is an incredible machine that protects, supports, and moves the human body. However, several diseases can limit its functionality, compromising patient quality of life. Designing novel pathological models would help to clarify the mechanisms driving such diseases, identify new biomarkers, and screen potential drug candidates. Miniaturized models in particular can mimic the structure and function of basic tissue units within highly controlled microenvironments, overcoming the limitations of traditional macroscale models and complementing animal studies, which despite being closer to the in vivo situation, are affected by species-specific differences. Here, we discuss the miniaturized models engineered over the past few years to analyze osteochondral and skeletal muscle pathologies, demonstrating how the rationale design of novel systems could provide key insights into the pathological mechanisms behind diseases of the musculoskeletal system.

\section{Introduction}

The average number of steps that each person should walk during a day is approximately equal to 10000 [1], which means that more than 200 million steps will be taken by a healthy individual between the age of 15 and 75 years. This outstanding performance is made possible by an efficient musculoskeletal system, in which bones, cartilage, muscles, tendons, and ligaments cooperate to protect, support, and move the human body. However, several pathologies can impair the structure and function of musculoskeletal tissues, including osteoarthritis (OA) [2] and muscular dystrophies [3].

Understanding the mechanisms underlying these diseases could help to generate novel diagnostic tools, develop new therapeutics, and identify optimal nutritional and physical programs to reduce the risk of their onset. In vivo and in vitro models represent an effective strategy to analyze specific features of biological processes and identify the main determinants of specific diseases. In vitro assays enable researchers to alter single parameters and analyze their effect on the whole system, which is technically challenging with animal studies. Moreover, despite reproducing the physiology of a living organism, animal models are affected by species-specific differences. Thus, over the past few years, several 3D in vitro models have been developed to study musculoskeletal tissue physiology, although they have rarely focused on specific diseases. A key aspect of these models is that embedded cells dynamically interact with the surrounding 3D extracellular matrix (ECM), and degrade matrix proteins so that they move and become organized into complex structures and polarize in response to physical stimuli [4]. In addition, cells in 3D conditions experience different mass transport phenomena and local forces compared with $2 \mathrm{D}$ conditions, involving changes in the spatial distribution of molecular gradients and the different organization of surface proteins [5]. Thus, the size of the system should be carefully designed to allow the proper diffusion of nutrients and oxygen. Computational simulations represent useful tools to predict mass transport phenomena and alter the size of the model accordingly [6]. Despite being closer to the in vivo environment, the design and development of 3D models is more time consuming, sample imaging can be compromised by light-scattering phenomena, and the recovery of cells can be limited by the presence of dense 3D matrices [7].

Recently, 3D miniaturized models have emerged as promising tools combining the advantages offered by traditional macroscale 3D models with the use of a limited amount of cells and reagents, real-time analysis of the whole sample through high-resolution imaging, and precise control of biochemical and biophysical stimuli [8]. Microfluidic strategies have been exploited to develop organ-on-a-chip systems, namely 3D miniaturized models that mimic the function of organ-specific tissue units, which can be used to analyze molecular and cellular events within biochemically and biophysically controlled microenvironments mimicking liver, lung, intestine, kidney, bone, skin, and heart $[9,10]$. Although not directly related to the study of musculoskeletal diseases, several research attempts have resulted in a lung-on-a-chip, which recapitulates the alveolar-capillary interface [11]. 
Despite increasing interest in the development of miniaturized models mimicking pathophysiological conditions, only a few groups have focused on the design of miniaturized models for musculoskeletal diseases. These models are characterized by peculiar features, according to the specific application and output for which they were designed. For instance, several systems have been developed in 3D to better mimic the architecture of the native tissue [12,13], although a few models are still based on 2D cultures [14]. The application of dynamic culture conditions is a critical aspect that provides in vivo-like biophysical stimuli to the microtissue to mimic pathophysiological conditions [15]. However, several models are still based on static microsystems, being more focused on the effect of biochemical stimuli [16] or the biomechanical properties of the ECM [17] in a specific musculoskeletal disease. In addition, miniaturized disease models have been developed using human cells [18], animal cells [19], monocultures [16], and co-cultures [20]. However, the use of more complex co-culture systems is mandatory when the pathological microenvironment involves interactions between multiple cell types [21]. Embedded human cells can be collected from a target primary tissue [17] or purchased as commercial cell lines [22]. In this framework, the identification of the most suitable cell source, including autologous cells [23] or induced pluripotent stem cells (iPSCs) [24], is crucial in establishing a functional model.

Most musculoskeletal miniaturized models reproduce physiological processes rather than pathological conditions. Indeed, the development of pathological models requires the design of specific biochemical, biophysical, and mechanical features characterizing a specific disease. Here, we highlight the miniaturized models developed over the past few years that mimic bone, osteochondral, and skeletal muscle diseases, demonstrating that the evolution of current systems could lead to not only a better understanding of biological mechanisms, but also the rationale design of novel platforms for diagnosis, drug screening, and disease treatment.

\section{Miniaturized models for bone and osteochondral diseases}

The use of miniaturized systems containing 2D or 3D bone-derived cultures represents a promising strategy for the development of in vitro models of orthopedic infections and bone diseases. Orthopedic infections, caused by bacteria forming antibiotic-resistant biofilms on implants, are a dominant cause of implant failure [25]. Lee et al. developed different microfluidic models of implant-associated infections. Specifically, they fabricated a microfluidic device integrating a Ti alloy and containing eight rectangular microchannels mounted on a microscope to real-time monitor the alterations induced by Staphylococcus epidermidis on the adhesion and proliferation of murine osteoblasts on the Ti surface [26]. They showed that the bacteria altered the microenvironment to create acidic conditions that impaired osteoblast viability and proliferation. The same microfluidic system was used to investigate the influence of different phenotypes of $S$. epidermidis on the ability of osteoblasts to selforganize into $3 \mathrm{D}$ bone-like structures [19]. Only the small colony variant of $S$. epidermidis induced osteoblast death, while the sessile biofilms and dead bacteria did not interfere with the formation of bone-like structures. This study demonstrated the possibility to generate a physiologically relevant model resembling the bone-biofilm-implant interfaces to study orthopedic infections. The same group also developed a microfluidic 3D bone model to examine the ability of different biomaterials to prevent bacterial infections [27]. Micropatterns containing antibiotic and/or biphasic calcium phosphate (BCP) nanoparticles dispersed in a poly(D,L-lactic-co-glycolic) acid (PLGA) matrix were inkjet-printed in a multichamber microfluidic device (Figure 1A). The micropatterns loaded with antibiotic and BCP immediately and completely killed S. epidermidis and enhanced the production of calcified matrix. Conversely, without antibiotic, bacteria rapidly proliferated, generating an acidic microenvironment that was detrimental to osteoblasts. This system was exploited to identify substrate combinations preventing bacterial infections while promoting calcified matrix deposition in dynamic conditions, emulating the nutrient and waste transport of the microcirculation. Together, the aforementioned studies demonstrate the potential of miniaturized models for the study and prevention of orthopedic infections.

Multiple myeloma (MM) is a plasma cell malignancy characterized by the formation of osteolytic bone lesions [28,29]. Based on the microfluidic devices designed by Lee and collaborators, Zhang et al. customized a 3D microfluidic system for the ex vivo maintenance of primary human multiple myeloma cells (MMCs) [21]. Specifically, a microfluidic multichamber device and a specific culture medium containing growth factors and cytokines from patients' plasma (Figure 1B), were used to recapitulate the complex tumor niche and to monitor the interactions between patient-derived MMCs and human fetal osteoblasts. The authors demonstrated, for the first time, that the direct co-culture of osteoblasts and MMCs and the dynamic culture in medium supplemented with patient-derived plasma sustained the ex vivo survival and proliferation of MMCs. This novel patient-specific MM model may provide new opportunities to explore this disease using primary cells as opposed to cell lines or complicated and costly patient-derived xenograft models. More recently, Zhang et al. also studied the interaction of osteoblasts and MMCs in a versatile well plate-based perfusion device assembled on a commercial polystyrene bottomless 96-well plate (Figure 1C) [13,22]. The ex vivo viability and proliferation of MMCs were preserved, confirming the determining influence of osteoblasts. Additionally, the authors demonstrated that the long-term survival of both osteoblasts and MMCs can be enhanced by controlling the flow rate, suggesting that these cells respond to mechanical stimuli [13]. In another study, the same well plate-based perfusion device was proposed to characterize osteoblast and MMC responses to drugs using conventional flow cytometry and plate-reading techniques [22]. From a clinical perspective, this technology provides a new avenue to test personalized therapies, screen new drugs without the need for animal models, and study the biology of MM as well as solid tumors, such as breast and prostate cancers, that metastasize to the bone through the same endosteal niche. 
Although bone diseases could in principle be investigated through the development of single-organ models, the analysis of osteochondral pathologies requires the development of more complex systems, given the interplay between cartilage and bone. In these systems, cartilage and bone should be integrated to mimic a functional osteochondral unit, possibly using multilayer biomaterials [30]. To mimic the whole articular joint, in vitro models should also include other elements, such as synovium and synovial fluid. Furthermore, other specific cell components, such as monocytes and/or macrophages, should be added when studying inflammatory diseases [31,32].

$\mathrm{OA}$ is a degenerative disease that eventually leads to the loss of joint function because of articular cartilage degradation and subchondral bone alterations [2,33]. The onset of this disease involves complex crosstalk between articular cartilage and subchondral bone [34,35]. In this context, the development of organotypic models resembling the osteochondral unit or the whole joint represents a promising approach to investigate OA pathogenesis and to test potential disease-modifying OA drugs (DMOADs), which are currently not available to patients [36]. Recently, Lin and colleagues developed a miniaturized model of the osteochondral unit fitting a multichamber bioreactor into a microfluidic base [15]. The microfluidic system was exploited to supply the chondral and osseous layer with two different media to create tissue-specific microenvironments and to achieve spatially defined differentiation of bone marrow mesenchymal stem cells (BMSCs). Subsequently, the microfluidic system was used to induce an OA-like response through the targeted treatment of the chondral or the osseous compartment with the proinflammatory cytokine interleukin-1 $\beta$ (IL-1 $\beta$ ). The results showed that the exposure of the osseous compartment to IL-1 $\beta$ resulted in a strong catabolic response in the chondral layer. This response was stronger than that seen when directly applying IL- $1 \beta$ to the chondral compartment, indicating the active communication between these two tissues. The same group further improved this model by integrating an osteochondral interface, comprising a BMSC-laden collagen hydrogel, between the chondral and the osseous compartments and by including hydroxyapatite in the osseous layer [37]. Moreover, to better resemble the joint environment, the authors proposed to incorporate the synovium component and endothelial cells into the system by lining the chondral layer with a BMSC-laden hydrogel to mimic the synovium and by perfusing endothelial cells within the osseous layer (Figure 2). Remarkably, this system is compatible with the application of physiological and pathological mechanical loads. Hence, in addition to its use as a high-throughput platform to test candidate DMOADs, this system will enable the investigation of the mechanisms of OA pathogenesis depending on both the excessive mechanical stimulation of the joint and the presence of catabolic and proinflammatory soluble factors.

\section{Discussion}

Physiological bone tissue is characterized by high vascularization and the continuous remodeling process determined by the balance between osteoblasts and osteoclasts. Furthermore, bone and cartilage in vivo are subjected to mechanical forces, such as pressure and shear, although those features are neglected by most in vitro models. The inclusion of a perfusable microvascular network in the bone compartment will be important to model diseases with an inflammatory component to reproduce the transendothelial migration of immune cells to the inflammation site. Furthermore, the presence of the endothelial component will be necessary when modeling diseases involving crosstalk between bone cells and endothelial cells. Also, none of the presented models showed the necessary complexity to mimic the bone-remodeling process. The incorporation of the osteoclastic component, as proposed in recent nonpathological bone models [38,39], represents an indispensable prerequisite when modeling diseases characterized by an imbalance in bone remodeling, such as MM, where malignant cells produce factors implicated in both osteoclast activation and osteoblast inhibition.

\section{Miniaturized models for skeletal muscle diseases}

Skeletal muscle comprises aligned, multinucleated myofibers containing actin and myosin organized in sarcomeres, the basic machinery for muscle contraction. Furthermore, neuromuscular junctions (NMJs) couple specialized neural cells (motor neurons) to myofibers and allow the transmission of signals causing myofibers contraction [40].

Skeletal muscle is able to regenerate following small injuries or acute damage; however, pathological conditions, such as genetic diseases, can alter this ability, leading to muscle atrophy. The most well-known muscular genetic disease, Duchenne muscular dystrophy (DMD), results from mutations in the dystrophin gene affecting the transmission of contraction from the sarcomeres to the ECM [3]. Miniaturized models of DMD have been described, including either in vitro models of diseased muscle [17,41] or microfluidic platforms for the investigation of pathological mechanisms at a cellular level [14,18]. A study by Serena and coworkers [17] was based on microgrooved soft polymeric substrates coated with laminin or fibronectin (Figure 3Ai). Micropatterning increased alignment and differentiation in both healthy and dystrophic myoblasts (Figure 3Aii), depending on the substrate stiffness and protein coating. The model was able to recapitulate specific pathological hallmarks, such as the loss of dystrophin expression in DMD compared with healthy myotubes, and the unchanged formation of sarcomeric units, a mechanism independent from dystrophin (Figure 3Aiii). The study by Vandenburgh and colleagues [41] had a different focus, describing a high-throughput drug-screening platform for compounds improving DMD muscle contractility. The platform comprised 3D muscle constructs engineered from mouse dystrophic myoblasts in collagen, which formed myofibers that were then subjected to electrical stimulation. Given the automated monitoring of muscle constructs contractility, it was found that only glucocorticoids improved DMD muscle contraction force. In addition to 3D miniaturized models of muscle, simpler 2D microfluidic devices have made it possible to investigate DMD pathological mechanisms at a cellular level, such as muscle regeneration after injury. 
Ferreira and collaborators [18] investigated the relation between basic fibroblast growth factor (FGF- $\beta$ )-mediated myoblast recruitment to injury site and different ECM compositions, representative of healthy and DMD microenvironments. Primary human myoblasts were cultured on laminin, fibronectin or collagen type I substrates in the presence of a FGF- $\beta$ gradient, highlighting how differences in ECM can modulate myoblasts chemotaxis and, thus, their recruitment to the injury site, hindering the physiological healing process of the muscle. Dysregulation of mechanosensitive ion channels also has a key role in the pathological mechanism of DMD [42] and, recently, a 2D microfluidic device was described that enabled the characterization of myoblast mechanotransduction operated by ionic channels subjected to interstitial stress [14]. C2C12 were cultured in a microfluidic chip coated with laminin or fibronectin and subjected to flow. The results obtained were in agreement with results from more traditional techniques, thus demonstrating the validity of the approach. Moreover, it was shown that mechanosensitive responses depended on protein substrate and medium composition, suggesting how the modifications of the pathological environment impact mechanotransduction in myoblasts.

Neuromuscular degenerative diseases, such as amyotrophic lateral sclerosis (ALS), represent another class of pathologies severely affecting skeletal muscle functions. Motor neuron physiology and development require a tightly regulated support of trophic factors, including glial cell-derived neurotrophic factor (GDNF), but their role in the establishment of degenerative pathologies has not yet been fully clarified [43]. In this context, Zahavi and collaborators [12] characterized how GDNF impacts motor neuron survival by co-culturing myoblasts and motor neurons within a microfluidic compartmentalized system mimicking a functional NMJ. Murine spinal cord explants were cultured in one compartment and extended axons through microgrooves, innervating myotubes in the other compartment (Figure 3Bi,ii). GDNF addition to the myotubes increased axon growth and motor neuron survival, but the same was not observed if GDNF was added to motor neuron bodies (Figure 3Biii), providing insights into the GDNF mechanism of action and further supporting its possible contribution to the pathogenesis of ALS.

Myasthenia gravis, characterized by muscle weakness, is an autoimmune disease whereby antibodies are generated and directed against specific neuromuscular synaptic molecules, such as the proteoglycan agrin [44]. A 2D microfluidic device was developed to study the effect of agrin on muscle fibers, restricting its delivery in defined regions of a micropatterned culture chamber [45]. C2C12 myoblasts were allowed to form myotubes in the microgrooves and were then stimulated with agrin, causing the stabilization of acetylcholine clusters, which are essential for the transmission of synaptic signals from motor neurons to muscle fibers, highlighting a new role for this molecule in the pathogenesis of myasthenia.

\section{Discussion}

Although producing interesting results, current models still lack important features that would enable them to fully characterize skeletal muscle pathologies. First, most miniaturized models are $2 \mathrm{D}$ systems, rather than 3D muscle tissues, and do not implement electrical stimulation to promote muscle contraction. Recently published macroscale muscle tissues showed that it is possible to obtain highly differentiated human myofibers that contract upon application of electrical stimulation and can be used to screen different drugs [46]. Translation of these techniques to the microscale would enable researchers to increase the control and throughput of the system [47]. Furthermore, to better characterize the response of pathological muscle models, the readout of important parameters such as muscle contractility, indicative of muscle health, should be added to the systems on the basis of similar apparatuses reported for cardiac models [48]. Lastly, novel findings indicate how the pathological muscular endothelium is an important element to be considered [49], although no vascularized pathological muscle model is currently available. However, both microfluidic [50] and macroscale [51] models of 3D vascularized muscle tissue have been recently described, suggesting forthcoming improvements for the in vitro modeling of neuromuscular diseases.

\section{Concluding remarks and outlook}

Significant advances have been made in the design of organ-specific models of the musculoskeletal system, including recently developed organs-on-a-chip to study bone metastases [50]. The choice between miniaturized and macroscale models is strictly dependent on the smallest tissue unit that is required to recapitulate and analyze an organ-specific disease. Based on the reverse engineering principle recently discussed by Ingber to describe microscale systems [52], the first step in the development of a functional pathological model is the identification of the minimum number of key elements that would mimic a specific disease. This issue implies a trade-off between complexity and progressive loss of control, which the designer must address to effectively recapitulate the pathological condition.

Remarkably, several reported miniaturized models are able to mimic features of musculoskeletal tissue structure and function, although they have never been exploited to study musculoskeletal diseases. For instance, the Asada group developed fascicle-like 3D structures comprising murine myoblasts within a microfluidic chip and measured their contractility upon electrical stimulation through an on-chip cantilever system [53]. This system could be improved with the use of iPSCs, as reported in similar studies in the field of cardiac diseases [54], leading to significant breakthroughs in the study of skeletal muscle disorders. Another promising microfluidic model was developed by Morimoto and colleagues, who generated functional neuromuscular junctions similar to their in vivo counterparts [55]. This system could find promising applications in the study of muscle disorders associated with the degeneration of the neuromuscular junctions, including ALS. In terms of the study of bone diseases, a 
microfluidic platform was fabricated as a potential in vitro screening tool for antiosteoporotic therapies, being able to deliver multiple drug concentrations to osteoblasts [56].

We expect that increasing relevance will be given to the structure and composition of matrix embedded within miniaturized models to finely match the mechanical properties of native tissues and promote physiological-like cell behaviors. 3D powder-printed microscale calcium phosphate was developed to generate bone substitutes with interconnected pores [57]. This technique could be optimized to generate calcium phosphate miniaturized models to analyze biological mechanisms and screen new therapeutics for bone diseases. In this context, vascularized bonemimicking miniaturized models have been recently designed to study the influence of oxygen tension and ECM composition on vascularization [58] and analyze osteoclast-mediated bone remodeling [39]. Finally, we envision that, over the next few years, implantable sensorized devices will be developed to monitor and treat musculoskeletal diseases, such as that recently described by the Langer group for the in vivo testing of anticancer agents on a living tumor [59].

In conclusion, only a few miniaturized pathological musculoskeletal models have been developed, although the number of systems recapitulating the physiology of specific tissue units is continuously increasing as well as their potential applications in the study of pathological conditions. We envision that recent advancements in microengineering will promote the design of novel organ-on-a-chip systems that can recapitulate complex cellular behaviors to study musculoskeletal diseases and represent useful high-content screening models bridging the gap between traditional high-throughput screening methods and animal models [60] to more effectively screen target drugs and provide promising candidates for clinical trials.

\section{Acknowledgments}

Funding from the Italian Ministry of Health and the Department of Defense of the USA is greatly acknowledged.

\section{References}

1 Tudor-Locke, C. and Bassett, D.R., Jr (2004) How many steps/day are enough? Preliminary pedometer indices for public health. Sports Med. $34,1-8$

2 Glyn-Jones, S. et al. (2015) Osteoarthritis. Lancet 386, 376-387

3 Cooper, S.T. and Head, S.I. (2015) Membrane injury and repair in the muscular dystrophies. Neuroscientist 21, 653-668

4 Schwartz, M.A. and Chen, C.S. (2013) Cell biology. Deconstructing dimensionality. Science 339, 402-404

5 Wolf, K. and Friedl, P. (2011) Extracellular matrix determinants of proteolytic and non-proteolytic cell migration. Trends Cell Biol. 21, 736744

6 Bersini, S. et al. (2016) Human in vitro 3D co-culture model to engineer vascularized bone-mimicking tissues combining computational tools and statistical experimental approach. Biomaterials 76, 157-172

7 Shamir, E.R. and Ewald, A.J. (2014) Three-dimensional organotypic culture: experimental models of mammalian biology and disease. Nat. Rev. Mol. Cell Biol. 15, 647-664

8 Shin, Y. et al. (2012) Microfluidic assay for simultaneous culture of multiple cell types on surfaces or within hydrogels. Nat. Protoc. 7, 12471259

9 Bhatia, S.N. and Ingber, D.E. (2014) Microfluidic organs-on-chips. Nat. Biotechnol. 32, 760-772

10 Uzel, S.G. et al. (2014) Microfabrication and microfluidics for muscle tissue models. Prog. Biophys. Mol. Biol. 115, 279-293

11 Huh, D. et al. (2012) A human disease model of drug toxicity-induced pulmonary edema in a lung-on-a-chip microdevice. Sci. Transl. Med. 4, 159ra147

12 Zahavi, E.E. et al. (2015) A compartmentalized microfluidic neuromuscular co-culture system reveals spatial aspects of GDNF functions. J. Cell Sci. 128, 1241-1252

13 Zhang, W. et al. (2015) Ex Vivo maintenance of primary human multiple myeloma cells through the optimization of the osteoblastic niche. PLoS ONE 10, e0125995

14 Kurth, F. et al. (2015) Transient receptor potential vanilloid 2-mediated shear-stress responses in C2C12 myoblasts are regulated by serum and extracellular matrix. FASEB J. 29, 4726-4737

15 Lin, H. et al. (2014) Stem cell-based microphysiological osteochondral system to model tissue response to interleukin-1beta. Mol. Pharm. 11, $2203-2212$

16 Nesmith, A.P. et al. (2014) Human airway musculature on a chip: an in vitro model of allergic asthmatic bronchoconstriction and bronchodilation. Lab Chip 14, 3925-3936

17 Serena, E. et al. (2010) Soft substrates drive optimal differentiation of human healthy and dystrophic myotubes. Integr. Biol. (Camb) 2, 193-201

18 Ferreira, M.M. et al. (2015) Microfluidic analysis of extracellular matrix-bFGF crosstalk on primary human myoblast chemoproliferation, chemokinesis, and chemotaxis. Integr. Biol. (Camb) 7, 569-579

19 Lee, J.H. et al. (2011) Microfluidic approach to create three-dimensional tissue models for biofilm-related infection of orthopaedic implants. Tissue Eng. Part C Methods 17, 39-48

20 Ibold, Y. et al. (2007) Development of a high-throughput screening assay based on the 3-dimensional pannus model for rheumatoid arthritis. J. Biomol. Screen. 12, 956-965

21 Zhang, W. et al. (2014) Patient-specific 3D microfluidic tissue model for multiple myeloma. Tissue Eng. Part C Methods 20, 663-670

22 Zhang, W. et al. (2015) Well plate-based perfusion culture device for tissue and tumor microenvironment replication. Lab Chip 15, 28542863

23 Klimczak, A. and Kozlowska, U. (2016) Mesenchymal stromal cells and tissue-specific progenitor cells: their role in tissue homeostasis. Stem Cells Int. 2016, 4285215

24 Ko, H.C. and Gelb, B.D. (2014) Concise review: drug discovery in the age of the induced pluripotent stem cell. Stem Cells Transl. Med. 3, $500-509$

25 Ribeiro, M. et al. (2012) Infection of orthopedic implants with emphasis on bacterial adhesion process and techniques used in studying bacterial-material interactions. Biomatter 2, 176-194

26 Lee, J.H. et al. (2010) Effects of Staphylococcus epidermidis on osteoblast cell adhesion and viability on a Ti alloy surface in a microfluidic co-culture environment. Acta Biomater. 6, 4422-4429

27 Lee, J.H. et al. (2012) Microfluidic 3D bone tissue model for high-throughput evaluation of wound-healing and infection-preventing biomaterials. Biomaterials 33, 999-1006

28 Giuliani, N. et al. (2006) Multiple myeloma bone disease: pathophysiology of osteoblast inhibition. Blood 108, 3992-3996

29 Roodman, G.D. (2009) Pathogenesis of myeloma bone disease. Leukemia 23, 435-441 
30 Lopa, S. and Madry, H. (2014) Bioinspired scaffolds for osteochondral regeneration. Tissue Eng. Part A 20, 2052-2076

31 Malemud, C.J. (2015) Biologic basis of osteoarthritis: state of the evidence. Curr. Opin. Rheumatol. 27, 289-294

32 Lopa, S. et al. (2015) Arthritic and non-arthritic synovial fluids modulate IL10 and IL1RA gene expression in differentially activated primary human monocytes. Osteoarthritis Cartilage 23, 1853-1857

33 Kingsbury, S.R. et al. (2014) Osteoarthritis in Europe: impact on health status, work productivity and use of pharmacotherapies in five European countries. Rheumatology (Oxford) 53, 937-947

34 Heinegard, D. and Saxne, T. (2011) The role of the cartilage matrix in osteoarthritis. Nat. Rev. Rheumatol. 7, 50-56

35 van der Kraan, P.M. and van den Berg, W.B. (2007) Osteophytes: relevance and biology. Osteoarthritis Cartilage 15, 237-244

36 Dubin, A. (2016) Managing osteoarthritis and other chronic musculoskeletal pain disorders. Med. Clin. North Am. 100, 143-150

37 Lozito, T.P. et al. (2013) Three-dimensional osteochondral microtissue to model pathogenesis of osteoarthritis. Stem Cell Res. Ther. 4 (Suppl. 1), S6

38 Papadimitropoulos, A. et al. (2011) A 3D in vitro bone organ model using human progenitor cells. Eur. Cell Mater. 21, 445-458; discussion 458

39 Bongio, M. et al. (2016) A 3D vascularized bone remodeling model combining osteoblasts and osteoclasts in a CaP nanoparticle-enriched matrix. Nanomedicine. Published online April 14, 2016. http://dx.doi.org/10.2217/nnm-2015-0021

40 Bursac, N. et al. (2015) Synergizing engineering and biology to treat and model skeletal muscle injury and disease. Annu. Rev. Biomed. Eng. $17,217-242$

41 Vandenburgh, H. et al. (2009) Automated drug screening with contractile muscle tissue engineered from dystrophic myoblasts. FASEB J. $23,3325-3334$

42 Brinkmeier, H. (2011) TRP channels in skeletal muscle: gene expression, function and implications for disease. Adv. Exp. Med. Biol. 704, $749-758$

43 Tovar, Y.R.L.B. et al. (2014) Trophic factors as modulators of motor neuron physiology and survival: implications for ALS therapy. Front. Cell. Neurosci. 8, 61

44 Gilhus, N.E. and Verschuuren, J.J. (2015) Myasthenia gravis: subgroup classification and therapeutic strategies. Lancet Neurol. 14, 10231036

45 Tourovskaia, A. et al. (2008) Localized acetylcholine receptor clustering dynamics in response to microfluidic focal stimulation with agrin Biophys. J. 95, 3009-3016

46 Madden, L. et al. (2015) Bioengineered human myobundles mimic clinical responses of skeletal muscle to drugs. Elife 4, e04885

47 Ramade, A. et al. (2014) Microfabrication of a platform to measure and manipulate the mechanics of engineered microtissues. Methods Cell. Biol. 121, 191-211

48 Grosberg, A. et al. (2012) Muscle on a chip: in vitro contractility assays for smooth and striated muscle. J. Pharmacol. Toxicol. Methods 65, $126-135$

49 Giannotta, M. et al. (2014) Targeting endothelial junctional adhesion molecule-A/ EPAC/ Rap-1 axis as a novel strategy to increase stem cell engraftment in dystrophic muscles. EMBO Mol. Med. 6, 239-258

50 Jeon, J.S. et al. (2015) Human 3D vascularized organotypic microfluidic assays to study breast cancer cell extravasation. Proc. Natl. Acad. Sci. U. S. A. 112, 214-219

51 Juhas, M. et al. (2014) Biomimetic engineered muscle with capacity for vascular integration and functional maturation in vivo. Proc. Natl. Acad. Sci. U. S. A. 111, 5508-5513

52 Ingber, D.E. (2016) Reverse engineering human pathophysiology with organs-on-chips. Cell 164, 1105-1109

53 Neal, D. et al. (2015) Mechanical characterization and shape optimization of fascicle-like 3D skeletal muscle tissues contracted with electrical and optical stimuli. Tissue Eng. Part A 21, 1848-1858

54 Wang, G. et al. (2014) Modeling the mitochondrial cardiomyopathy of Barth syndrome with induced pluripotent stem cell and heart-on-chip technologies. Nat. Med. 20, 616-623

55 Morimoto, Y. et al. (2013) Three-dimensional neuron-muscle constructs with neuromuscular junctions. Biomaterials 34, 9413-9419

56 Nason, F. et al. (2011) Design of microfluidic devices for drug screening on in-vitro cells for osteoporosis therapies. Microelectronic Engineering 88, XXX-YYY

57 Meininger, S. et al. (2015) Strength reliability and in vitro degradation of three-dimensional powder printed strontium-substituted magnesium phosphate scaffolds. Acta Biomater. YY, XXX-YYY

58 Bersini, S. et al. (2015) Human in vitro 3D co-culture model to engineer vascularized bone-mimicking tissues combining computational tools and statistical experimental approach. Biomaterials $76,157-172$

59 Jonas, O. et al. (2015) An implantable microdevice to perform high-throughput in vivo drug sensitivity testing in tumors. Sci Transl Med 7 , $284 \mathrm{ra} 257$

60 Vandenburgh, H. (2010) High-content drug screening with engineered musculoskeletal tissues. Tissue Eng. Part B Rev. 16, 55-64

Figure 1. Miniaturized models for bone diseases. (A) A poly-dimethyl-siloxane (PDMS)-based microfluidic device with eight culture chambers integrating inkjet-printed micropatterns and housed in an environmental chamber for real-time microscopy. Each chamber has an elongated hexagonal shape (12 mm long, $6 \mathrm{~mm}$ wide, and 0.2 $\mathrm{mm}$ deep). (B) Schematic representation of a 3D microfluidic system for the ex vivo maintenance of multiple mieloma cells. (i) Bone marrow-multiple mieloma cells (BMMC) were harvested from patients and frozen in liquid nitrogen. ii) Human osteoblasts were cultured for 4 days in the hexagonal-shaped chambers of the microfluidic device to obtain an ossified tissue before addition of BMMC. (iii) BMMC were thawed and fluorescently labeled. (iv) Fluorescent-labeled BMMC were seeded into the culture chambers. (v) After 21 days, the interactions between BMMC and 3D ossified tissue and BMMC proliferation were monitored by real-time microscopy and flow cytometry, respectively. (C) Schematics of design features and fabrication steps of a 96-well plate-based perfusion device. Two wells were used to produce one culture chamber with one inlet and one outlet, resulting in 48 chambers available for cell culture. The outlet chamber was used to direct the effluent stream to exit through the device top so that the bottom device could be accessible for plate reading and microscopy. The culture device comprised three 1 mm-thick PDMS layers to provide a fluidic channel $(2 \mathrm{~mm}$ thick, $6 \mathrm{~mm}$ wide) between the inlet and outlet chambers and to anchor the transperent polycarbonate (PC) membranes (1$\mathrm{mm}$ pore size). The bottom of the device was sealed with a $1.2 \mathrm{~mm}$-thick glass layer. The device had an overall thickness of $13.5 \mathrm{~mm}$, fitting a plate reader. PDMS plugs were also placed at the top of the culture wells to enable placement of cells and biomaterials into the culture wells at various time points during culture. Reproduced, with permission, from [27] (A), [21] (B), and [22] (C)

Figure 2. Schematic of the workflow and milestones in the development of a functional miniaturized osteochondral model. A chondral compartment (cc) and an osseous compartment (oc) are joined by an intermediate layer (mc, mesenchymal construct) and supplied by specific medium streams through the upper medium conduit (uc) and the lower medium conduit (Ic). Endothelial cells (ec) are included in the platform ( $p$ ) to generate a vascularized osseous compartment and human fibroblasts (hf) are used to generate a synovial compartment. Analytical methods compatible with the platform are listed in the 'Methods Key' in the figure. Reproduced, with permission, from [37]

Figure 3. Title. (A) (i) Schematic of protein coating by microcontact printing. Proteins (red) are transferred to the soft hydrogel (light blue) by means of a poly-dimethylsiloxane (PDMS) mold (white) with $75 \mathrm{~mm}$-wide and $100 \mathrm{~mm}$-spaced grooves. (ii) Phase-contrast micrograph showing highly aligned human myoblasts seeded on microgrooved hydrogels. (iii) Representative immunofluorescent staining for myosin heavy chain (green) and $\alpha$-actinin (red) of dystrophic myotubes, showing the formation of sarcomeres after 7 days of culture (nuclei are stained blue with DAPI). (B) (i) Fluorescent and phase-contrast images of axons (green) growing from GFP transfected spinal explants (upper row), through microgrooves (middle), and finally innervating myotubes (lower row). Scale bars: 50 mm. (ii) High-magnification fluorescence images showing the formation of neuromuscular junctions (NMJs). Axons are stained in green, synapsin in white, and postsynaptic acetylcholine clusters 
in myotubes in BTX red staining, in a representative axon-muscle contact area. Scale bar: $5 \mathrm{~mm}$. (iii) Fluorescence imaging of the neuronal and muscular compartments, showing the effects of glial cell-derived neurotrophic factor (GDNF) addition in the muscular compartment. Violet staining highlights the increased content of phosphorylated Akt (a well-characterized prosurvival effector) in motor neuron bodies with the addition of GDNF. Scale bars: 50 mm. Reproduced, with permission, from [17] (A) and [12] (B). 
A)

C)

Flow
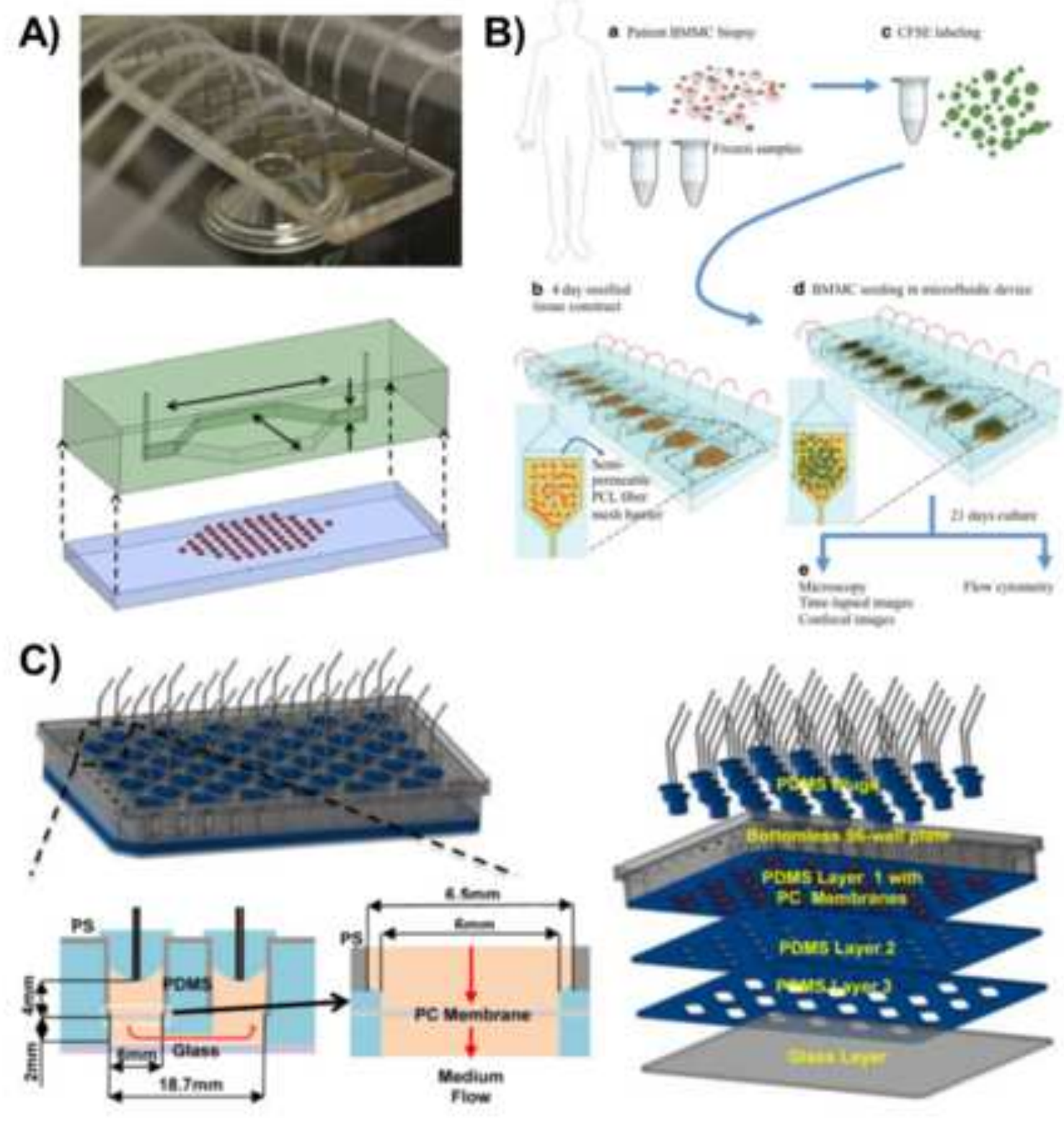


\section{Figure 2}

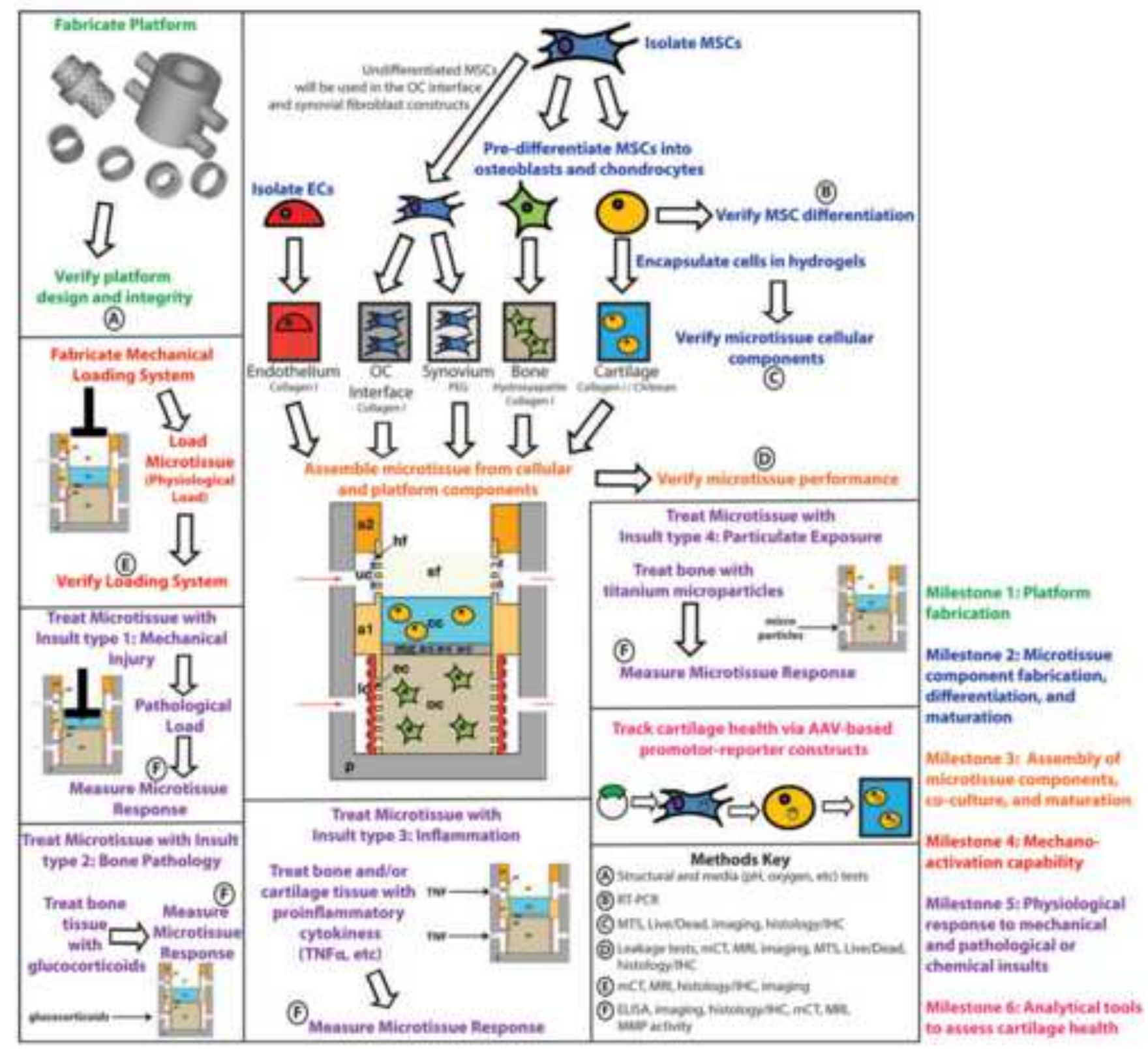


A)

i)

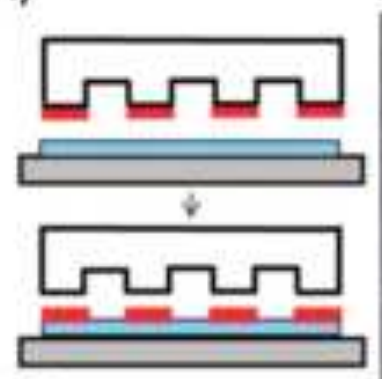

iii)

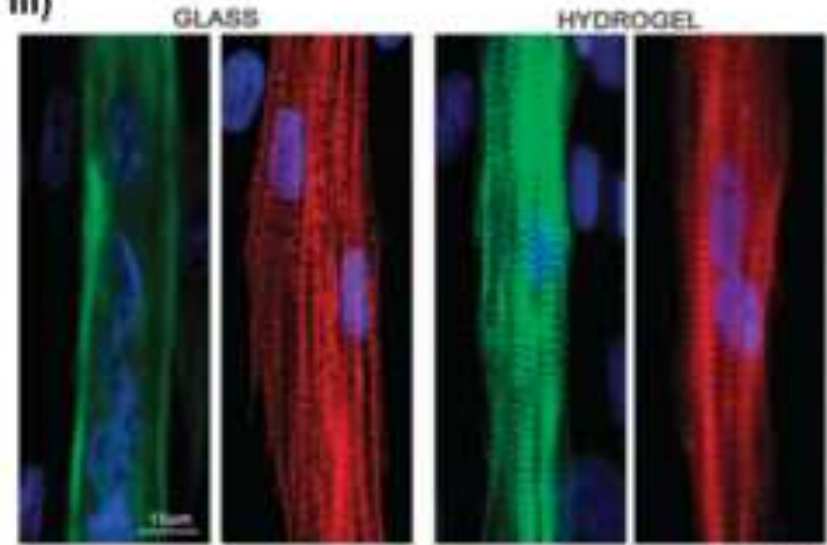

ํํำ

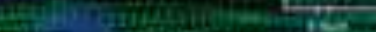

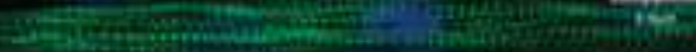

ii)

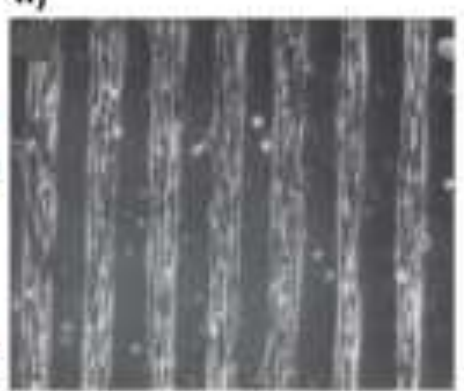

Hropoce
B)

i)
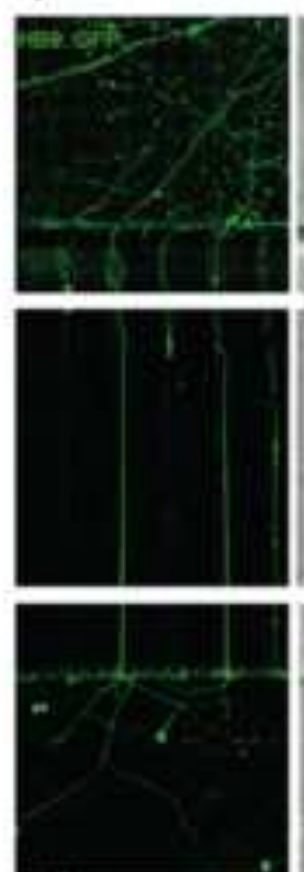

iii)

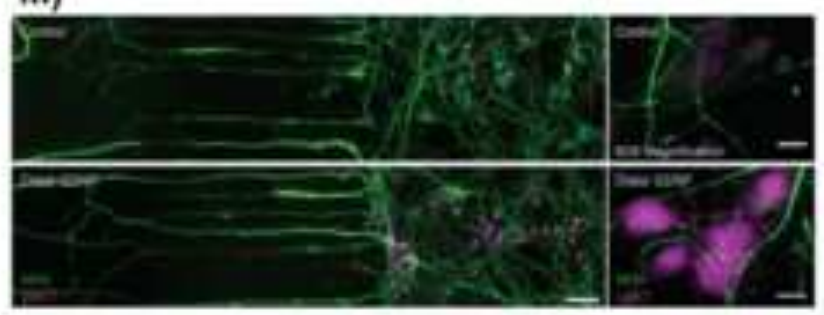

ii)
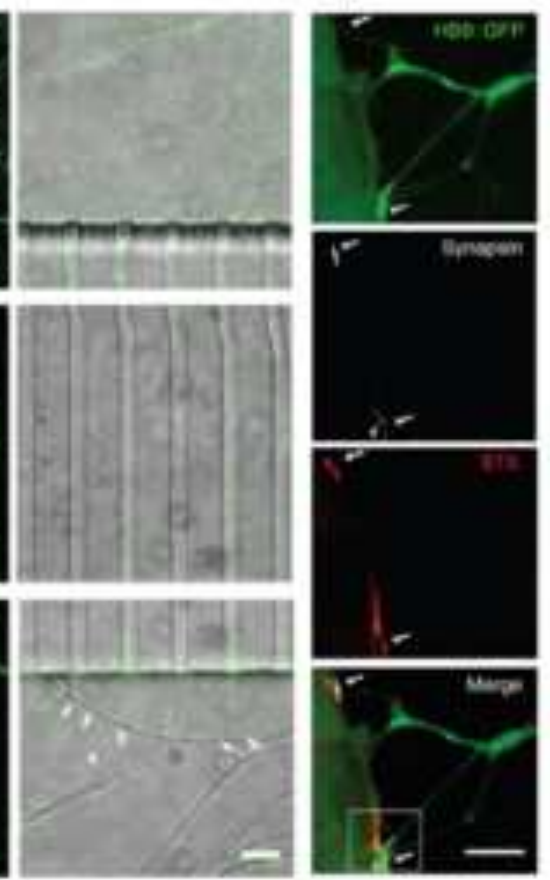

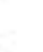

N

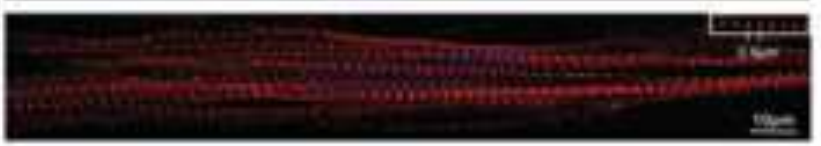

